\title{
Dual-isotope cardiac SPECT: the Twin Peaks Saga in nuclear cardiology
}

\author{
C. Marcassa, MD, a and O. Zoccarato, $\mathrm{PhD}^{\mathrm{b}}$ \\ a Department of Cardiology, ICS Maugeri, Institute for Clinical Care and Research (IRCCS), \\ Scientific Institute of Veruno, Novara, Italy \\ b Department of Nuclear Medicine, ICS Maugeri, Institute for Clinical Care and Research (IRCCS), \\ Scientific Institute of Veruno, Novara, Italy
}

Received Apr 6, 2017; accepted Apr 6, 2017

doi:10.1007/s12350-017-0900-5

\section{See related article, pp. 1692-1704}

The use of two different isotopes to study the myocardium with SPECT imaging is almost as old as nuclear cardiology itself. ${ }^{1}$ The majority of the experience was done with T1201 and 99mTc-labeled tracers, ${ }^{2}$ with T1201 injected at rest and $99 \mathrm{mTc}$-perfusion tracers injected at peak stress, although different isotope and tracer combinations have been also investigated. ${ }^{3,4}$ Two separate sets of SPECT images are acquired, one for each isotope or condition.

Simultaneous dual-isotope SPECT imaging allows for the concomitant evaluation of the processes targeted by the two radioisotopes (e.g., viability with thallium and ischemia with $99 \mathrm{mTc}$ tracers or viability with thallium and neuroadrenergic damage with I-123MIBG). Moreover, imaging two isotopes in one scan obviously reduces the probability of errors induced by image misalignment, and increases patient comfort.

A potential problem with this technique is the downscatter of the higher peak into the lower one (the crosstalk effect, defined as the contamination in one isotope's energy window due to the detection of the photons from the other isotope), because of the interactions of the photons in the patient, collimator-detector system, or the limited energy resolution of conventional Anger cameras, which could result in image degradation.

\footnotetext{
Reprint requests: C. Marcassa, Department of Cardiology, Salvatore Maugeri Foundation, Institute for Clinical Care and Research (IRCCS), Scientific Institute of Veruno, Novara, Italy cmarcassa@fsm.it

J Nucl Cardiol 2018;25:1705-7.

$1071-3581 / \$ 34.00$

Copyright (C) 2017 American Society of Nuclear Cardiology.
}

In the case of simultaneous T1201 and Tc-99m, Tc$99 \mathrm{~m}$ photons scattered in the body and lead X-rays created in the collimator will be detected in the Tl-201 energy window. Several methods for dual-isotope imaging have been suggested to correct for the downscatter, including estimating and subtracting it prior to reconstruction or reconstruction-based compensation. This can be done either in the spatial domain using crosstalk or using measurements in multiple energy windows. ${ }^{5-10}$

The majority of these correction methods is based on estimating the downscatter by blurring and scaling projection data acquired from an intermediate scatter window (three-window method). However, they cannot accurately take into account the patient dependency of the downscatter. Furthermore, a downscatter estimate directly based on measured projections is often noisy, which will add noise to the downscatter-corrected image. ${ }^{11}$

In the case of I-123 and Tc-99m imaging, however, the photopeak energies are close together, 140 and $159 \mathrm{keV}$, respectively: the primary photons of I-123 and its downscattered photons would be detected in the Tc$99 \mathrm{~m}$ energy window, and the primary photons of Tc$99 \mathrm{~m}$ may be contained in I-123 window as well, and the correction for crosstalk is more difficult.

Simultaneous ${ }^{99 \mathrm{~m}} \mathrm{Tc} /{ }^{123} \mathrm{I}$ imaging was applied in brain studies. $^{12,13}$

Kumita et al evaluated the simultaneous assessment of Tc-99m sestamibi and I-123-BMIPP to assess myocardial perfusion and metabolism. ${ }^{14}$ Du et al assessed the feasibility of simultaneous dual ${ }^{99 \mathrm{~m}} \mathrm{Tc} /{ }^{123} \mathrm{I}$ imaging to assess myocardial perfusion and innervation using Siemens IQ-SPECT. ${ }^{15}$ In this study, the compensation of the estimated crosstalk was included in an iterative reconstruction process, and the method was then validated with data from both Monte Carlo simulation and physical phantom experiments. 
The latest generation of gamma cameras uses novel semiconductor cadmium zinc telluride (CZT) detector technology and might be considered as the real technical milestone in nuclear cardiac imaging in the last 20 years; they provide a superior energy resolution, scatter fraction, sensitivity, count rate capability, and spatial resolution, when compared to conventional gamma cameras.

The advantages of the increased energy resolution of cadmium zinc telluride (CZT) cameras (e.g., at $140 \mathrm{keV} 6 \%$ vs. $9.25 \%$ of conventional cameras) in dual-isotope imaging $\left({ }^{99 \mathrm{~m}} \mathrm{Tc}\right.$ and $\left.{ }^{123} \mathrm{I}\right)$ were recently assessed. In 69 patients prospectively evaluated with ${ }^{201} \mathrm{Tl} /{ }^{123} \mathrm{I}$-MIBG SPECT to investigate viability/innervation mismatch, the scatter correction was applied using the three energy window method. ${ }^{16}$

In a first experience using a dynamic cardiac phantom, Blaire et al documented that the assessment of LVEF and regional motion with $123 \mathrm{I}$ or $99 \mathrm{mTc}$ and the new CZT cameras was comparable using different energy windows and acquisition types (single vs. dual). 17

In another well-controlled phantom experimental study, appearing in this issue of the Journal, ${ }^{18}$ these authors investigated the feasibility and potential limitations of simultaneous $99 \mathrm{mTc} / \mathrm{I} 123$ dual-isotope acquisitions to evaluate the myocardial wall, with the two CTZ systems currently available. This type of phantom evaluation is mandatory when evaluating a new technique with such potential clinical applications as well as such potentially serious errors and artifacts.

In the experiments they performed, Blaire et al. documented that reconstructed wall activities of $99 \mathrm{mTc}$ (but not 123I) were significantly affected by the acquisition modality (single vs. dual) using both cameras. Image contrast using 123I and DNM 530c or both 123I and $99 \mathrm{mTc}$ with D-SPECT was also influenced by the acquisition type.

In the interpretation of the results, however, two major issues should be taken into account. First, besides sharing the same detector technology, the two tomographic systems are substantially different. The DNM 530c is equipped with a multiple pinhole collimator and 19 stationary CZT detectors that are able to simultaneously acquire 19 cardiac views, whereas the D-SPECT system adopts 9 mobile blocks of pixelated CZT detectors coupled with a wide-angle square-hole tungsten collimator, arranged so that each detector's pixel is seen from a single hole of the collimator; during the tomographic acquisition this system acquires 120 projections by each block (a total of 1080 projections). Moreover, the iterative reconstruction algorithms and the post-reconstruction filters are also different. Finally, in addition to the different architecture and reconstruction algorithms, it should be considered that different corrections were applied to different acquisition types/camera combinations: while scatter, crosstalk, and tailing effect were applied using the D-SPECT for dual but not for single isotope acquisitions, no correction at all was applied with DNM 530c acquisitions.

Nevertheless, despite the above-mentioned differences, as final result the defect and mismatch size (that are the two major data we are looking for) were not influenced by the type of acquisition.

Myocardial perfusion imaging is a cornerstone in the diagnostic and prognostic evaluation of patients with coronary artery disease. More recently, the assessment of cardiac neuroadrenergic damage with I-123-MIBG is increasing in popularity in the identification of patients with severe left ventricular dysfunction at higher risk of events. The simultaneous assessment of both information, perfusion and innervation, minimizing the likelihood of errors induced by image misalignment would provide a more accurate detection of mismatch areas, that are viable but denervated, thought to be involved in the genesis of arrhythmias.

Blaire et al are to be appreciated for their effort. Back in 1990, in David Lynch serial drama the FBI Special Agent Dale Cooper was sent to unveil the secrets of Twin Peaks; in the third millennium the new CZT cameras might open new horizons in the use of multi-peak acquisitions in myocardial SPECT imaging, although additional phantom studies and clinical validation are necessary before a full clinical deployment.

\section{Disclosure}

The authors have indicated that they have no financial conflict of interest.

\section{References}

1. Fukuda H, Nakamura K, Nemoto Y, Kiyota T, Sakuyama K, Shibamoto S, Ochi H, Onoyama Y, Ikuno Y. The dual energy myocardial SPECT of 99mTc-PYP and 201TlCl for diagnosis of acute myocardial infarction. Kaku Igaku. 1985;22:1545-9.

2. Berman DS, Kiat H, Friedman JD, Wang FP, van Train K, Matzer L, Maddahi J, Germano G. Separate acquisition rest thallium-201/ stress technetium-99m sestamibi dual-isotope myocardial perfusion single-photon emission computed tomography: a clinical validation study. J Am CollCardiol. 1993;22(5):1455-64.

3. Kropp J. Myocardial scintigraphy with iodine-123 phenylpentadecanoic acid and thallium-201 in patients with coronary artery disease: a comparative dual-isotope study. Eur J Nucl Med. 1993;20(4):364-6.

4. Maeno M, Ishida Y, Shimonagata T, Hayashida K, Toyama T, Hirose Y, Nagata M, Miyatake K, Uehara T, Nishimura T. The significance of 201T1/123I MIBG (metaiodobenzylguanidine) mismatched myocardial regions for predicting ventricular 
tachycardia in patients with idiopathic dilated cardiomyopathy. KakuIgaku. 1993;30(10):1221-9.

5. Buvat I, Hapdey S, Benali H, Todd-Pokropek A, Di Paola R. Spectral factor analysis for multi-isotope imaging in nuclear medicine. Lect Notes Comput Sc. 1999;1613:442-7.

6. Hannequin P, Mas J, Germano G. Photon energy recovery for crosstalk correction in simultaneous Tc- $99 \mathrm{~m} / \mathrm{Tl}-201$ imaging. J Nucl Med. 2000;41(4):728-36.

7. Ichihara T, Ogawa K, Motomura N, Kubo A, Hashimoto S. Compton scatter compensation using the triple-energy window method for single-isotope and dual-isotope spect. J Nucl Med. 1993;34(12):2216-21.

8. Knesaurek K. A new dual-isotope convolution cross-talk correction method-a Tl-201 Tc-99m Spect Cardiac Phantom Study. Med Phys. 1994;21(10):1577-83.

9. Moore SC, English RJ, Syravanh C, Tow DE, Zimmerman RE, Chan KH, et al. Simultaneous Tc-99m/Tl-201 imaging using energy-based estimation of the spatial distributions of contaminant photons. IEEE Trans Nucl Sci. 1995;42(4):1189-95.

10. Wells RG, Celler A, Harrop R. Analytical calculations of crosstalk for dual-isotope SPECT projections. IEEE Trans Nucl Sci. 2000;47(3):1202-9.

11. Yang JT, Yamamoto K, Sadato N, Tsuchida T, Takahashi N, Hayashi N, Yonekura Y, Ishii Y. Clinical value of triple-energy window scatter correction in simultaneous dual-isotope singlephoton emission tomography with 123I-BMIPP and 201Tl. Eur J Nucl Med. 1997;24(9):1099-106.

12. El Fakhri G, Moore SC, Maksud P, Aurengo A, Kijewski MF. Absolute activity quantitation in simultaneous $123 \mathrm{I} / 99 \mathrm{mTc}$ brain SPECT. J Nucl Med. 2001;42:300-8.
13. El Fakhri G, Habert M, Maksud P, Kas A, Malek Z, Kijewski MF, Lacomblez L. Quantitative simultaneous 99mTc-ECD/123I-FPCIT SPECT in Parkinson's disease and multiple system atrophy. Eur J Nucl Med Mol Imaging. 2006;33:87-92.

14. Kumita SI, Cho K, Nakajo H, Toba M, Kijima T, Mizumura S, Kumazaki T, Sano J, Munakata K, Kishida H, Takano T. Simultaneous assessment of Tc-99m-sestamibi and I-123-BMIPP myocardial distribution in patients with myocardial infarction: evaluation of left ventricular function with ECG-gated myocardial SPECT. Ann Nucl Med. 2000;14(6):453-539.

15. Du Y, Bhattacharya M, Frey EC. Simultaneous Tc-99m/I-123 dual radionuclide myocardial perfusion/innervation imaging using siemens IQ-SPECT with SMARTZOOM collimator. Phys Med Biol. 2014;59(11):2813-28.

16. D'estanque E, Hedon C, Lattuca B, Bourdon A, Benkiran M, Verd A, Roubille F, Mariano-Goulart D. Optimization of a simultaneous dual-isotope 201T1/123I-MIBG myocardial SPECT imaging protocol with a CZT camera for trigger zone assessment after myocardial infarction for routine clinical settings: are delayed acquisition and scatter correction necessary? J Nucl Cardiol. 2016. doi:10.1007/s12350-016-0524-1.

17. Blaire T, Bailliez A, Bouallegue FB, Bellevre D, Agostini D, Manrique A. Left ventricular function assessment using 123I/ $99 \mathrm{mTc}$ dual-isotope acquisition with two semi-conductor cadmium-zinc-telluride (CZT) cameras: a gated cardiac phantom study. EJNMMI Phys. 2016;3(1):27.

18. Blaire T, Bailliez A, Ben Bouallegue F, Bellevre D, Agostini D, Manrique A. First assessment of simultaneous dual isotope (123I/ 99mTc) cardiac SPECT on two different CZT cameras: a phantom study. J Nucl Cardiol 2017. doi:10.1007/s12350-017-0841-z. 